1 Hacettepe Journal of Mathematics and Statistics

$\bigcap$ Volume 47 (5) (2018), $1120-1127$

\title{
Maximal accretive singular quasi-differential operators
}

\author{
Pembe Ipek ${ }^{* \dagger}$ and Zameddin I. Ismailov ${ }^{\ddagger}$
}

\begin{abstract}
In this paper firstly all maximal accretive extensions of the minimal operator generated by a first order linear singular quasi-differential expression in the weighted Hilbert space of vector-functions on right semi-axis are described. Later on, the structure of spectrum set of these extensions has been researched.
\end{abstract}

Keywords: Accretive operator, Quasi-differential operator, Spectrum.

Mathematics Subject Classification (2010): 47A10, 47B25

Received : 21.03.2017 Accepted : 19.06.2017 Doi : 10.15672/HJMS.2017.488

\section{Introduction}

It is known that a linear closed densely defined operator $T: D(T) \subset H \rightarrow H$ in Hilbert space $H$ is called accretive(dissipative) if for all $f \in D(T)$ the inequality $R e<T f, f>_{H} \geq 0\left(\operatorname{Im}<T f, f>_{H} \geq 0\right)$ is satisfied. Also it is called maximal accretive(maximal dissipative) if it is accretive(dissipative) and does not have any proper accretive(dissipative) extension [3], [1]. The class of accretive operators is an important class of non-selfadjoint operators in the operator theory. Note that the spectrum set of accretive operators lies in right half-plane.

The maximal accretive extensions and their spectral analysis of the minimal operator generated by regular differential-operator expression in Hilbert space of vector-functions defined in one finite interval case have been studied by V.V. Levchuk [4].

This work is organised as follows: In Section 3, all maximal accretive extensions of the minimal operator generated by a linear singular quasi-differential operator expression in the weighted Hilbert spaces of the vector functions defined at right semi-axis are examined. In Section 4, the structure of the spectrum of these type extensions has been investigated.

*Institute of Natural Sciences, Karadeniz Technical University, 61080, Trabzon, Turkey, Email: ipekpembe@gmail.com

${ }^{\dagger}$ Corresponding Author.

${ }^{\ddagger}$ Department of Mathematics, Karadeniz Technical University, 61080, Trabzon, Turkey, Email: zameddin.ismailov@gmail.com 


\section{Statement of the problem}

Let $H$ be a separable Hilbert space and $a \in \mathbb{R}$. Moreover assumed that $\alpha:(a, \infty) \rightarrow$ $(0, \infty), \alpha \in C(a, \infty)$ and $\alpha^{-1} \in L^{1}(a, \infty)$. In the weighted Hilbert space $L_{\alpha}^{2}(H,(a, \infty))$ of $H$ - valued vector-functions defined on the right semi-axis consider the following linear first order quasi-differential expression with operator coefficient

$$
l(u)=(\alpha u)^{\prime}+A u,
$$

where $A: H \rightarrow H$ is a selfadjoint operator with condition $A \geq 0$.

By a standard way the minimal $L_{0}$ and maximal $L$ operators corresponding to quasidifferential expression $l\left({ }^{\cdot}\right)$ in $L_{\alpha}^{2}(H,(a, \infty))$ can be defined (see [2]). In this case the minimal operator $L_{0}$ is accretive, but it is not maximal in $L_{\alpha}^{2}(H,(a, \infty))$.

The main goal of this work is to describe of all maximal accretive extensions of the minimal operator $L_{0}$ in terms of boundary condition in $L_{\alpha}^{2}(H,(a, \infty))$. Secondly, the structure of the spectrum set of these extensions will be investigated.

\section{Description of maximal accretive extensions}

Note that in similar way the minimal operator $L_{0}^{+}$generated by a quasi-operator expression

$$
l^{+}(v)=-(\alpha v)^{\prime}+A v
$$

can be defined in $L_{\alpha}^{2}\left(H,(a, \infty)\right.$ ) (see [2]). In this case the operator $L^{+}=\left(L_{0}\right)^{*}$ in $L_{\alpha}^{2}(H,(a, \infty))$ is called the maximal operator generated by $l^{+}(\cdot)$. It is clear that $L_{0} \subset L$ and $L_{0}^{+} \subset L^{+}$.

If $\widetilde{L}$ is any maximal accretive extension of the minimal operator $L_{0}$ in $L_{\alpha}^{2}(H,(a, \infty))$ and $\widetilde{M}$ is corresponding extension of the minimal operator $M_{0}$ generated by a quasidifferential expression

$$
m(u)=i(\alpha u)^{\prime}
$$

in $L_{\alpha}^{2}(H,(a, \infty))$, then it is clear that

$$
\begin{aligned}
\widetilde{L} u & =(\alpha u)^{\prime}(t)+A u(t) \\
& =i\left(-i(\alpha u)^{\prime}\right)(t)+A u(t) \\
& =i(-\widetilde{M})(t)+A u(t) \\
& =i(-(\operatorname{Re} \widetilde{M}+i \operatorname{Im} \widetilde{M})) u(t)+A u(t) \\
& =(\operatorname{Im} \widetilde{M}) u(t)-i(\operatorname{Re} \widetilde{M}) u(t)+A u(t) \\
& =[(\operatorname{Im} \widetilde{M})+A] u(t)-i(\operatorname{Re} \widetilde{M}) u(t) .
\end{aligned}
$$

Therefore

$$
(\operatorname{Re} \widetilde{L})=(\operatorname{Im} \widetilde{M})+A
$$

On the other hand it is clear that

$$
(\operatorname{Re} \widetilde{L})=(\operatorname{Im} \widetilde{M})+A=\operatorname{Im}(\widetilde{M}+A) .
$$

Hence to describe all maximal accretive extension of the minimal operator $L_{0}$ in $L_{\alpha}^{2}(H,(a, \infty))$ it is sufficiently to describe all maximal dissipative extensions of the minimal operator $S_{0}$ generated by quasi-differential expression

in $L_{\alpha}^{2}(H,(a, \infty))$.

$$
s(u)=i(\alpha u)^{\prime}+A u
$$

Furthermore, we will denote the maximal operator generated by the quasi-differential expression $s(\cdot)$ in $L_{\alpha}^{2}(H,(a, \infty))$ by $S$. 
In this section, we will investigate the general representation of all maximal dissipative extensions of the minimal operator $S_{0}$ in $L_{\alpha}^{2}(H,(a, \infty))$ by using Calkin-Gorbachuk method. Let us prove the following proposition.

3.1. Lemma. The deficiency indices of the minimal operator $S_{0}$ in $L_{\alpha}^{2}(H,(a, \infty))$ are given in the form

$$
\left(n_{+}\left(S_{0}\right), n_{-}\left(S_{0}\right)\right)=(\operatorname{dim} H, \operatorname{dim} H) .
$$

Proof. For the simplicity of calculations, we will take $A=0$. It is clear that the general solutions of differential equations

$$
i\left(\alpha u_{ \pm}\right)^{\prime}(t) \pm i u_{ \pm}(t)=0, t>a
$$

in $L_{\alpha}^{2}(H,(a, \infty))$

$$
u_{ \pm}(t)=\frac{1}{\alpha(t)} \exp \left(\mp \int_{a}^{t} \frac{d s}{\alpha(s)}\right) f, f \in H, t>a
$$

From these representations, we have

$$
\begin{aligned}
\left\|u_{+}\right\|_{L_{\alpha}^{2}(H,(a, \infty))}^{2} & =\int_{a}^{\infty}\left\|u_{+}(t)\right\|_{H}^{2} d t \\
& =\int_{a}^{\infty}\left\|\frac{1}{\alpha(t)} \exp \left(-\int_{a}^{t} \frac{d s}{\alpha(s)}\right) f\right\|_{H}^{2} \alpha(t) d t \\
& =\int_{a}^{\infty} \frac{1}{\alpha(t)} \exp \left(-2 \int_{a}^{t} \frac{d s}{\alpha(s)}\right) d t\|f\|_{H}^{2} \\
& =\int_{a}^{\infty} \exp \left(-2 \int_{a}^{t} \frac{d s}{\alpha(s)}\right) d\left(\int_{a}^{t} \frac{d s}{\alpha(s)}\right)\|f\|_{H}^{2} \\
& =\frac{1}{2}\left(1-\exp \left(-2 \int_{a}^{\infty} \frac{d s}{\alpha(s)}\right)\right)\|f\|_{H}^{2}<\infty .
\end{aligned}
$$

Consequently $n_{+}\left(S_{0}\right)=\operatorname{dim} \operatorname{ker}(S+i E)=\operatorname{dim} H$. 
On the other hand, it is clear that for any $f \in H$,

$$
\begin{aligned}
\left\|u_{-}\right\|_{L_{\alpha}^{2}(H,(a, \infty))}^{2} & =\int_{a}^{\infty}\left\|u_{-}(t)\right\|_{H}^{2} d t \\
& =\int_{a}^{\infty}\left\|\frac{1}{\alpha(t)} \exp \left(\int_{a}^{t} \frac{d s}{\alpha(s)}\right) f\right\|_{H}^{2} \alpha(t) d t \\
& =\int_{a}^{\infty} \frac{1}{\alpha(t)} \exp \left(2 \int_{a}^{t} \frac{d s}{\alpha(s)}\right) d t\|f\|_{H}^{2} \\
& =\int_{a}^{\infty} \exp \left(2 \int_{a}^{t} \frac{d s}{\alpha(s)}\right) d\left(\int_{a}^{t} \frac{d s}{\alpha(s)}\right)\|f\|_{H}^{2} \\
& =\frac{1}{2}\left(\exp \left(2 \int_{a}^{\infty} \frac{d s}{\alpha(s)}\right)-1\right)\|f\|_{H}^{2}<\infty
\end{aligned}
$$

It follows from that $n_{-}\left(S_{0}\right)=\operatorname{dim} \operatorname{ker}(S-i E)=\operatorname{dim} H$. This completes the proof of the theorem.

Consequently, the minimal operator $S_{0}$ has a maximal dissipative extension (see [1]).

In order to describe these extensions, we need to obtain the space of boundary values.

3.2. Definition. [1] Let $\mathfrak{H}$ be any Hilbert space and $S: D(S) \subset \mathfrak{H} \rightarrow \mathfrak{H}$ be a closed densely defined symmetric operator in the Hilbert space $\mathfrak{H}$ having equal finite or infinite deficiency indices. A triplet $\left(\mathbf{H}, \gamma_{1}, \gamma_{2}\right)$, where $\mathbf{H}$ is a Hilbert space, $\gamma_{1}$ and $\gamma_{2}$ are linear mappings from $D\left(S^{*}\right)$ into $\mathbf{H}$, is called a space of boundary values for the operator $S$ if for any $f, g \in D\left(S^{*}\right)$

$$
<S^{*} f, g>_{\mathfrak{H}}-<f, S^{*} g>_{\mathfrak{H}}=<\gamma_{1}(f), \gamma_{2}(g)>_{\mathbf{H}}-<\gamma_{2}(f), \gamma_{1}(g)>_{\mathbf{H}}
$$

while for any $F_{1}, F_{2} \in \mathbf{H}$, there exists an element $f \in D\left(S^{*}\right)$ such that $\gamma_{1}(f)=F_{1}$ and $\gamma_{2}(f)=F_{2}$.

\subsection{Lemma. Define}

$$
\begin{aligned}
\gamma_{1}: D(S) \rightarrow H, \gamma_{1}(u) & =\frac{1}{\sqrt{2}}((\alpha u)(\infty)-(\alpha u)(a)) \text { and } \\
\gamma_{2}: D(S) \rightarrow H, \gamma_{2}(u) & =\frac{1}{i \sqrt{2}}((\alpha u)(\infty)+(\alpha u)(a)), u \in D(S) .
\end{aligned}
$$

Then the triplet $\left(H, \gamma_{1}, \gamma_{2}\right)$ is a space of boundary values of the minimal operator $S_{0}$ in $L_{\alpha}^{2}(H,(a, \infty))$. 
Proof. For any $u, v \in D(S)$

$$
\begin{aligned}
& <S u, v>_{L_{\alpha}^{2}(H,(a, \infty))}-<u, S v>_{L_{\alpha}^{2}(H,(a, \infty))} \\
= & <i(\alpha u)^{\prime}+A u, v>_{L_{\alpha}^{2}(H,(a, \infty))}-<u, i(\alpha v)^{\prime}+A v>_{L_{\alpha}^{2}(H,(a, \infty))} \\
= & <i(\alpha u)^{\prime}, v>_{L_{\alpha}^{2}(H,(a, \infty))}-<u, i(\alpha v)^{\prime}>_{L_{\alpha}^{2}(H,(a, \infty))} \\
= & \int_{a}^{\infty}<i(\alpha u)^{\prime}(t), v(t)>_{H} \alpha(t) d t-\int_{a}^{\infty}<u(t), i(\alpha v)^{\prime}(t)>_{H} \alpha(t) d t \\
= & i\left[\int_{a}^{\infty}<(\alpha u)^{\prime}(t),(\alpha v)(t)>_{H} d t+\int_{a}^{\infty}<(\alpha u)(t),(\alpha v)^{\prime}(t)>_{H} d t\right] \\
= & i \int_{a}^{\infty}<(\alpha u)(t),(\alpha v)(t)>_{H}^{\prime} d t \\
= & i\left[<(\alpha u)(\infty),(\alpha v)(\infty)>_{H}-<(\alpha u)(a),(\alpha v)(a)>_{H}\right] \\
= & <\gamma_{1}(u), \gamma_{2}(v)>_{H}-<\gamma_{2}(u), \gamma_{1}(v)>_{H} .
\end{aligned}
$$

Now for any given elements $f, g \in H$, we can find the function $u \in D(S)$ such that $\gamma_{1}(u)=\frac{1}{\sqrt{2}}((\alpha u)(\infty)-(\alpha u)(a))=f$ and $\gamma_{2}(u)=\frac{1}{i \sqrt{2}}((\alpha u)(\infty)+(\alpha u)(a))=g$.

From this, we obtain

$$
(\alpha u)(\infty)=(i g+f) / \sqrt{2} \text { and }(\alpha u)(a)=(i g-f) / \sqrt{2} .
$$

If we choose the function $u(\cdot)$ in following form

$$
u(t)=\frac{1}{\alpha(t)}\left(1-e^{a-t}\right)(i g+f) / \sqrt{2}+\frac{1}{\alpha(t)} e^{a-t}(i g-f) / \sqrt{2},
$$

then it is clear that $u \in D(S)$ and $\gamma_{1}(u)=f, \gamma_{2}(u)=g$.

The following result can be established by using the method given in [1].

3.4. Theorem. If $\widetilde{S}$ is a maximal dissipative extension of the minimal operator $S_{0}$ in $L_{\alpha}^{2}(H,(a, \infty))$, then it is generated by the differential-operator expression $s(\cdot)$ and boundary condition

$$
(\alpha u)(a)=K(\alpha u)(\infty)
$$

where $K: H \rightarrow H$ is a contraction operator. Moreover, the contraction operator $K$ in $H$ is determined uniquely by the extension $\widetilde{S}$, i.e. $\widetilde{S}=S_{K}$ and vice versa.

Proof. It is known that each maximal dissipative extension $\widetilde{S}$ of the minimal operator $S_{0}$ is described by the differential-operator expression $s(\cdot)$ and the boundary condition

$$
(V-E) \gamma_{1}(u)+i(V+E) \gamma_{2}(u)=0,
$$

where $V: H \rightarrow H$ is a contraction operator. Therefore from Lemma 3.3, we obtain

$$
(V-E)((\alpha u)(\infty)-(\alpha u)(a))+(V+E)((\alpha u)(\infty)+(\alpha u)(a))=0, u \in D(\widetilde{S}) .
$$

From this, it implies that

$$
(\alpha u)(a)=-V(\alpha u)(\infty) .
$$

Choosing $K=-V$ in last boundary condition, we have

$$
(\alpha u)(a)=K(\alpha u)(\infty)
$$


From this theorem and the note mentioned above, it implies the validity of the following result.

3.5. Theorem. Each maximal accretive extension $\widetilde{L}$ of the minimal operator $L_{0}$ is generated by linear singular quasi-differential expression $l(\cdot)$ and boundary condition

$$
(\alpha u)(a)=K(\alpha u)(\infty)
$$

where $K: H \rightarrow H$ is a contraction operator such that this operator is determined uniquely by the extension $\widetilde{L}$, i.e. $\widetilde{L}=L_{K}$ and vice versa.

\section{The spectrum of the maximal accretive extensions}

In this section the structure of the spectrum set of the maximal accretive extensions of the minimal operator $L_{0}$ in $L_{\alpha}^{2}(H,(a, \infty))$ will be researched.

4.1. Theorem. The spectrum of any maximal accretive extension $L_{K}$ has the form

$$
\begin{aligned}
\sigma\left(L_{K}\right)= & \left\{\lambda \in \mathbb{C}: \lambda=\left(\int_{a}^{\infty} \frac{d s}{\alpha(s)}\right)^{-1}\left(\ln \left(|\mu|^{-1}\right)+\operatorname{iarg}(\bar{\mu})+2 n \pi i\right),\right. \\
& \left.\mu \in \sigma\left(\operatorname{Kexp}\left(-A \int_{a}^{\infty} \frac{d s}{\alpha(s)}\right)\right), n \in \mathbb{Z}\right\} .
\end{aligned}
$$

Proof. Consider the following problem to get the spectrum of the extension $L_{K}$, i.e.

$$
L_{K}(u)=\lambda u+f, \lambda \in \mathbb{C}, \lambda_{r}=\operatorname{Re} \lambda \geq 0
$$

Then we have

$$
\begin{aligned}
& (\alpha u)^{\prime}(t)+A u(t)=\lambda u(t)+f(t), t>a \\
& (\alpha u)(a)=K(\alpha u)(\infty)
\end{aligned}
$$

The general solution of the last differential equation

$$
(\alpha u)^{\prime}(t)=\frac{1}{\alpha(t)}(\lambda E-A)(\alpha u)(t)+f(t), t>a
$$

is

$$
\begin{aligned}
u(t ; \lambda) & =\frac{1}{\alpha(t)} \exp \left((\lambda E-A) \int_{a}^{t} \frac{d s}{\alpha(s)}\right) f_{\lambda} \\
& -\frac{1}{\alpha(t)} \int_{t}^{\infty} \exp \left((\lambda E-A) \int_{s}^{t} \frac{d \tau}{\alpha(\tau)}\right) f(s) d s, f_{\lambda} \in H, t>a .
\end{aligned}
$$


In this case

$$
\begin{aligned}
& \left\|\frac{1}{\alpha(t)} \exp \left((\lambda E-A) \int_{a}^{t} \frac{d s}{\alpha(s)}\right) f_{\lambda}\right\|_{L_{\alpha}^{2}(H,(a, \infty))}^{2} \\
= & \int_{a}^{\infty}\left\|\frac{1}{\alpha(t)} \exp \left((\lambda E-A) \int_{a}^{t} \frac{d s}{\alpha(s)}\right) f_{\lambda}\right\|_{H}^{2} \alpha(t) d t \\
= & \int_{a}^{\infty}<\frac{1}{\alpha(t)} \exp \left((\lambda E-A) \int_{a}^{t} \frac{d s}{\alpha(s)}\right) f_{\lambda}, \frac{1}{\alpha(t)} \exp \left((\lambda E-A) \int_{a}^{t} \frac{d s}{\alpha(s)}\right) f_{\lambda}>_{H} \alpha(t) d t \\
= & \int_{a}^{\infty} \frac{1}{\alpha(t)} \exp \left(2 \lambda_{r} \int_{a}^{t} \frac{d s}{\alpha(s)}\right)<\exp \left(-A \int_{a}^{t} \frac{d s}{\alpha(s)}\right) f_{\lambda}, \exp \left(-A \int_{a}^{t} \frac{d s}{\alpha(s)}\right) f_{\lambda}>_{H} d t \\
= & \int_{a}^{\infty} \frac{1}{\alpha(t)} \exp \left(2 \lambda_{r} \int_{a}^{t} \frac{d s}{\alpha(s)}\right)\left\|\exp \left(-A \int_{a}^{t} \frac{d s}{\alpha(s)}\right) f_{\lambda}\right\|_{H}^{2} d t \\
\leq & \int_{a}^{\infty} \frac{1}{\alpha(t)} \exp \left(2 \lambda_{r} \int_{a}^{t} \frac{d s}{\alpha(s)}\right) d t\left\|f_{\lambda}\right\|_{H}^{2} \\
= & \frac{1}{2 \lambda_{r}}\left(\exp \left(2 \lambda_{r} \int_{a}^{\infty} \frac{d s}{\alpha(s)}\right)-1\right)\left\|f_{\lambda}\right\|_{H}^{2}<\infty
\end{aligned}
$$

and

$$
\begin{aligned}
&\left\|-\frac{1}{\alpha(t)} \int_{t}^{\infty} \exp \left((\lambda E-A) \int_{s}^{t} \frac{d \tau}{\alpha(\tau)}\right) f(s) d s\right\|_{L_{\alpha}^{2}(H,(a, \infty))}^{2} \\
&= \int_{a}^{\infty}\left\|\frac{1}{\alpha(t)} \int_{t}^{\infty} \exp \left((\lambda E-A) \int_{s}^{t} \frac{d \tau}{\alpha(\tau)}\right) f(s) d s\right\|_{H}^{2} \alpha(t) d t \\
&= \int_{a}^{\infty} \frac{1}{\alpha(t)}\left\|\int_{t}^{\infty} \exp \left((\lambda E-A) \int_{s}^{t} \frac{d \tau}{\alpha(\tau)}\right) f(s) d s\right\|_{H}^{2} d t \\
&= \int_{a}^{\infty} \frac{1}{\alpha(t)}\left\|\int_{t}^{\infty} \exp \left(\lambda E \int_{s}^{t} \frac{d \tau}{\alpha(\tau)}\right)\left[\exp \left(-A \int_{s}^{t} \frac{d \tau}{\alpha(\tau)}\right) f(s)\right] d s\right\|_{H}^{2} d t \\
&=\int_{a}^{\infty} \frac{1}{\alpha(t)}\left\|\int_{t}^{\infty} \exp \left(\left(\lambda_{r}+i \lambda_{i}\right) \int_{s}^{t} \frac{d \tau}{\alpha(\tau)}\right)\left[\exp \left(-A \int_{s}^{t} \frac{d \tau}{\alpha(\tau)}\right) \frac{1}{\sqrt{\alpha(s)}}(\sqrt{\alpha(s)} f(s))\right] d s\right\|_{H}^{2} d t
\end{aligned}
$$




$$
\begin{aligned}
& \leq \int_{a}^{\infty} \frac{1}{\alpha(t)}\left(\int_{a}^{\infty} \frac{1}{\alpha(s)} \exp \left(2 \lambda_{r} \int_{s}^{t} \frac{d \tau}{\alpha(\tau)}\right) d s\right)\left(\int_{a}^{\infty} \alpha(s)\|f\|_{H}^{2} d s\right) d t \\
& \leq \int_{a}^{\infty} \frac{1}{\alpha(t)}\left(\int_{a}^{\infty} \frac{1}{\alpha(s)} \exp \left(2 \lambda_{r} \int_{a}^{\infty} \frac{d \tau}{\alpha(\tau)}\right) d s\right)\|f\|_{L_{\alpha}^{2}(H,(a, \infty))}^{2} \\
& =\exp \left(2 \lambda_{r} \int_{a}^{\infty} \frac{d \tau}{\alpha(\tau)}\right)\left(\int_{a}^{\infty} \frac{d s}{\alpha(s)}\right)^{2}\|f\|_{L_{\alpha}^{2}(H,(a, \infty))}^{2}<\infty .
\end{aligned}
$$

Hence $u(\cdot, \lambda) \in L_{\alpha}^{2}(H,(a, \infty))$ for $\lambda \in \mathbb{C}, \operatorname{Re} \lambda \geq 0$.

Furthermore from boundary condition, we get

$$
\left(E-K \exp \left((\lambda E-A) \int_{a}^{\infty} \frac{d s}{\alpha(s)}\right)\right) f_{\lambda}=\int_{a}^{\infty} \exp \left((\lambda E-A) \int_{s}^{a} \frac{d \tau}{\alpha(\tau)}\right) f(s) d s .
$$

Therefore in order to obtain $\lambda \in \sigma\left(L_{K}\right)$ the necessary and sufficient condition is

$$
\exp \left(-\lambda \int_{a}^{\infty} \frac{d s}{\alpha(s)}\right)=\mu \in \sigma\left(K \exp \left(-A \int_{a}^{\infty} \frac{d s}{\alpha(s)}\right)\right) \text {. }
$$

Hence

$$
-\lambda \int_{a}^{\infty} \frac{d s}{\alpha(s)}=\ln |\mu|+\operatorname{iarg} \mu+2 m \pi i, m \in \mathbb{Z},
$$

that is, $\lambda=\left(\int_{a}^{\infty} \frac{d s}{\alpha(s)}\right)^{-1}\left(\ln \left(|\mu|^{-1}\right)+\operatorname{iarg}(\bar{\mu})+2 n \pi i\right), n \in \mathbb{Z}, \mu \in \sigma\left(K \exp \left(-A \int_{a}^{\infty} \frac{d s}{\alpha(s)}\right)\right)$.

Example. All maximal accretive extensions $L_{r}$ of the minimal operator $L_{0}$ generated by a differential expression

$$
l(u)=\left(t^{\alpha} u(t, x)\right)^{\prime}+A u(t, x), \alpha>1
$$

in Hilbert space $L_{t^{\alpha}}^{2}((0,1) \times(1, \infty))$ in terms of boundary conditions are described by the following form

$$
\left(t^{\alpha} u(t, x)\right)(1)=r\left(t^{\alpha} u(t, x)\right)(\infty), 0<r<1,0<x<1,
$$

where

$$
A: L^{2}(0,1) \rightarrow L^{2}(0,1), A v(x)=x v(x)
$$

Moreover, the spectrum of such extensions is

$$
\sigma\left(L_{r}\right)=\left\{\lambda \in \mathbb{C}: \lambda=(1-\alpha)\left(\ln \left(|\mu|^{-1}\right)+\operatorname{iarg}(\bar{\mu})+2 n \pi i\right), \mu \in \sigma\left(\operatorname{rexp}\left(\frac{A}{\alpha-1}\right)\right), \quad n \in \mathbb{Z}\right\} .
$$

\section{References}

[1] Gorbachuk, V.I and Gorbachuk, M.I. Boundary Value Problems for Operator Differential Equations, Kluwer Academic Publisher, Dordrecht, 1991.

[2] Hörmander, L. On the Theory of General Partial Differential Operators, Acta Math. 94 (1), 161-248, 1955.

[3] Kato, T. Perturbation Theory for Linear Operators, Springer-Verlag Inc., New York, 1966, 592 pp.

[4] Levchuk, V.V. Smooth Maximally Dissipative Boundary-Value Problems for a Parabolic Equation in a Hilbert Space, Ukrainian Math. J. 35 (4), 502-507, 1983. 